improvement for patients suffering from rheumatic diseases, only in rare cases a therapy-free remission is achieved. In most cases stopping of treatment results in disease relapse. Apparently, components of the immune system are refractory to conventional immunosuppression and can drive the inflammation. Experimental and clinical evidence suggests that cells of the immunological memory persist despite immunosuppression and if pathogenic play a major role in the chronification of the disease. In particular long-lived memory plasma cells secreting autoantibodies represent a major therapeutic challenge. Once generated, they are not subject to physiological and even conventional therapeutic immune regulation. Their elimination may be prerequisite to curative therapies. A detailed understanding the lifestyle of long-lived memory plasma cells will be important to address this cell type therapeutically.

Disclosure of Interest: None declared

DOI: 10.1136/annrheumdis-2017-eular.7257

\section{SP0087 HOW ANTIGEN PRESENTING CELLS CAN BE TURNED INTO TOLEROGENIC CELLS}

J.D. Isaacs. Insitute of Cellular Medicine, Newcastle University, Newcastle upon Tyne, United Kingdom

Antigen presenting cells (APCs) lay at the heart of all immune responses. Whereas we generally consider APCs as cells that stimulate immune reactivity, they are also critically important for avoiding autoreactivity. Thus in health our tissues are patrolled by cells such as immature dendritic cells, which downregulate responses to self-antigens. Corruption of this process is a central factor in autoimmunity.

A number of groups have developed methods to generate "tolerogenic" antigen presenting cells, that mimic the cells which regulate self-tolerance in health. It is hypothesised that administration of such cells, loaded with autoantigens, to patients with autoimmune disease should be able to overcome autoreactivity and re-establish immune regulation. Our own group has developed a therapeutic approach based upon autologous tolerogenic dendritic cells, which we derive from circulating peripheral blood monocytes. Unlike conventional mature DC, which produce IL-12p70 and other pro-inflammatory cytokines, tolDC produce no IL-12p70 but high levels of IL-10. They deviate naïve T-cells towards an IL-10-producing, anti-inflammatory phenotype and induce hyporesponsiveness in memory T-cells. In mixed cultures they dominate mature, pro-inflammatory DC and down-regulate T-cell activation. Their phenotype is stable in the presence of pro-inflammatory stimuli. Equivalent murine toIDC switch off collagen-induced arthritis, with immune deviation from IL-17 to IL-10 production by CD4+ T cells and a reduction in type II collagen-specific $T$ cell responses.

In a phase 1 trial (AuToDeCRA), we demonstrated that these cells are safe when administered into a recently inflamed target knee joint of patients with inflammatory arthritis. However, in that safety study we were unable to demonstrate a tolerogenic effect in vivo. Furthermore, we have reason to believe that administered cells may remain in the target joint, whereas a disease-modifying effect is likely to require migration to secondary lymphoid tissues. Moving forwards we are designing a study that will address the optimal administration route for tolDC, based on a technique to track the cells in vivo and to measure their effect on autoreactivity. Disclosure of Interest: None declared

DOI: 10.1136/annrheumdis-2017-eular.7253

\section{THURSDAY, 15 JUNE 2017}

\section{What to do about comorbidity?}

\section{SP0088 NEW DRUGS, BUT STILL COMORBIDITY}

A. Häkkinen. Health Sciences, University of Jyväskylä, 40014 Jyväskylä University, Finland

As the population ages the simultaneous presence of multiple pathological conditions in the form of comorbidity and multimorbidity is more a rule than an exception. Comorbidity is reported in 35 to $80 \%$ of all ill people (Taylor et al. 2010). Comorbidity and multimorbidity are challenging researchers, clinicians and policy makers as these persons require more frequent appointments and hospitalizations and are at a greater risk for drug interactions, disability and mortality (Slater 2011).

Although numerous chronic disease prevention strategies and treatment guidelines have been developed, they mainly address single conditions and ignore the presence of co-existing conditions (van der Noyen 2016). Especially physical activity in its different forms has numerous preventive and curative effects in most of the diseases in addition to drugs. These benefits are such as increased muscle force and aerobic capacity, maintenance of bone and cardiovascular health, decreased inflammation and pain, improved function and well-being.

Studies reveal that more than $80 \%$ of rheumatoid arthritis (RA) patients carry two or more comorbid conditions (Krisnan et al. 2005). However, according to the QUEST-RA study (5,235 patients from 21 countries), only $14 \%$ of all patients reported to perform physical exercise at least 3 times weekly. Physical inactivity was associated with female sex, older age, obesity, comorbidity, disability, disease activity, pain and fatigue (Sokka et al. 2008). Traditionally, patients with RA were advised to limit physical exercises due to a fear that exercises might increase disease activity and be harmful for joints but more recent studies show that they benefit from exercise (Baillet et al. 2012).

Compared to RA, osteoarthritis (OA) is more common with prevalence of $\sim 150$ million people world-wide. In OA comorbidity rates vary between $68-85 \%$ in different studies. The most frequently occurring co-morbidities are diabetes, hypertension, cardiovascular disorders, obesity and back pain. De Rooij et al. (2016) have developed tailored exercise therapy for knee OA and comorbidity. In their study during the 20 -week program $76 \%$ of the participants needed adaptations to frequency, type, intensity or duration of exercise sessions. In addition, $96 \%$ needed education and coaching related to comorbidities.

In our study group-based strength and balance training for two years was offered for community-dwelling participants aged $>75$ years. The results showed that those who did not start in the group had more comorbidities, lower cognition, higher sedative load, higher risk of malnutrition, and poorer self-reported health than those who started in the gym. Despite of multimorbidity and hospital admissions, many older adults were capable of long-term regular training (Aartolahti et al. 2015).

With multimorbidity multi-drug therapies are common and they increase the risk of side effects. Exercise is also beneficial for health and it should be considered as a non-pharmacological drug. As for any other drugs, individual dosing of exercise is very important as well.

References:

[1] Aartolahti E et al. Gerontol Geriatr.2015;61:452-7.

[2] Baillet A et al. Rheumatology 2012;51:519-527.

[3] de Rooij M et al. Arthritis Care Res. 2016 Aug 26.

[4] Krishnan E et al Ann Rheum Dis. 2005;64:1350-2.

[5] Slater M, BMC Public Health 2011:11:77.

[6] Sokka T et al Arthritis Rheum. 2008:15;59:42-50.

[7] Taylor A et al. BMC Public Health 2010;10:718.

[8] van der Zee-Neuen A Arthritis Care \& Research 2016:68,1823-183.

Disclosure of Interest: None declared

DOI: 10.1136/annrheumdis-2017-eular.7061

\section{SP0089 HOW TO PREVENT AND TREAT CARDIOVASCULAR COMORBIDITY WITH EXERCISE?}

\section{H. Dagfinrud. Diakonhjemmet Hospital, Oslo, Norway}

Low cardiorespiratory fitness is a strong predictor of cardiovascular disease and all-cause mortality in healthy people as well as in patient groups. Unfit individuals have twice the risk of death from all causes, and tailored exercise is important to improve fitness.

It is well established that patients with inflammatory rheumatic diseases have increased risk for cardiovascular disease compared with healthy population, and it is therefore particularly important that these patients benefit from the risk-reducing effect of exercise. Exercise has traditionally been recommended as part of the treatment for patients with rheumatic diseases, but exercise programs has mainly focused on improving mobility and reducing pain. Further, patients with active disease has been recommended to exercise with low intensity. To increase cardiorespiratory fitness, however, high intensity exercise is needed. It is therefore encouraging that recent studies show that patients with active rheumatic disease tolerate intensive cardiorespiratory- and strength exercises and can benefit from such health-enhancing training. Recent research in this field will be presented and discussed.

Disclosure of Interest: None declared

DOI: 10.1136/annrheumdis-2017-eular.7287

\section{SP0090 COMORBIDITY-ADAPTED EXERCISE FOR PATIENTS WITH KNEE OSTEOARTHRITIS}

M. De Rooij. READE, Center for Rehabilitation and Rheumatology, Amsterdam, Netherlands

Exercise therapy is a key intervention in the management of patients with knee $\mathrm{OA}^{1}$. However, comorbidity is present in 68 to $85 \%$ of patients with OA (e.g. cardiac disease, diabetes type 2 , obesity $)^{2,3}$. Comorbidity interferes with exercise therapy. In clinical practice, comorbidity is a frequent reason to exclude patients from exercise therapy. If accepted into an exercise program, both therapists and patients tend to reduce exercise intensity to a level unlikely to be effective, because of fear of aggravating symptoms of the comorbid disease. Further, the effect of exercise therapy in patients with knee OA and severe comorbidity is not known. Patients with unstable medical conditions, precluding safe participation in an exercise program, are excluded from clinical trials. In view of the effectiveness of exercise therapy in knee OA and the high prevalence of comorbidity, there is a great need for comorbidity-related adaptations to exercise therapy. In this lecture a strategy (i3-S strategy) will be presented on how to develop comorbidity-related adaptations to exercise therapy in an index disease (e.g osteoarthritis) ${ }^{4}$. According this strategy we have developed a tailored exercise program for patients with knee $\mathrm{OA}$ and comorbidity. Subsequently, to evaluate the efficacy of the tailored exercise program for patients with knee OA and comorbidity (cardiac disease, diabetes type 2, COPD and obesity (body mass index $>30 \mathrm{~kg} / \mathrm{m}^{2}$ ) a randomized controlled trial $(n=126)$ was performed in a secondary care setting. The results showed that tailored exercise therapy greatly improved physical functioning, reduced pain and 